\title{
Influências de Parâmetros Acústicos na Educação do Século XXI: Contexto da Universidade Federal da Paraíba (UFPB) em 2015
}

\author{
Influences of Acoustics Parameters in the XXI Century Education: Context \\ of the Universidade Federal da Paraíba (UFPB) in 2015
}

Influencias de Parámetros Acústicos en la Educación del Siglo XXI: Contexto de la Universidade Federal da Paraíba (UFPB) em 2015

André Vieira Sonoda ${ }^{1}$

\begin{abstract}
Resumo
Apresentação de pesquisa implementada no âmbito da Universidade Federal da Paraíba em 2015 com o intuito de analisar níveis de pressão sonora em pontos de convivência no Campus I da Instituição, além de possíveis influências destes contextos acústicos no desempenho acadêmico discente. Objetiva chamar atenção para a possibilidade de influências negativas diretas e indiretas destes ambientes sonoros no desempenho acadêmico. Entre os subsídios teóricos para a elaboração do presente trabalho constam análises bibliográficas, análises da atuação discente no projeto, dados obtidos na primeira fase da pesquisa, além de técnicas de observação participante, entrevistas, gráficos e tabelas. Espera-se que este trabalho possa contribuir para o aprofundamento e a ampliação das discussões em torno do tema, visando iniciativas pedagógicas e Institucionais que evitem influências do tipo no desempenho acadêmico.
\end{abstract}

Palavras-Chave: Análises Acústicas, Análises de Pressão Sonora, UFPB, Desempenho Acadêmico.

\begin{abstract}
Research presentation implemented in the Universidade Federal da Paraíba (UFPB) in 2015 in order to identify levels of sound pressure in coexistence points at Campus I of the institution beyond influences of these acoustic contexts in academic performance. Has objective of call attention to the possibility of negative influences direct and indirect of these sound environments on education. Among the theoretical subsidies for this work were bibliographic analyzes, analyzes of student actuation on the project, data from the first phase of the research, in addition to participant observation techniques, interviews, charts and tables. It is expected that this work can contribute to the development and expansion of discussions around the issue, seeking to educational and Institutional initiatives that prevent influences like this on academic performance.
\end{abstract}

Keywords: Acoustic analysis, Sound Pressure Analysis, UFPB, Academic Performance.

\section{Resumen}

Presentación de investigación realizada en la Universidad Federal de Paraíba en 2015 con el objetivo de analizar los niveles de presión sonora en puntos de convivencia en el Campus I de la Institución, así como las posibles influencias de estos contextos acústicos en el rendimiento académico de los estudiantes. Tiene como objetivo llamar la atención sobre la posibilidad de influencias negativas directas e indirectas de estos paisajes sonoros en el rendimiento académico. Entre las bases teóricas para la preparación de este trabajo incluyó el análisis de la

\footnotetext{
1 Mestre em Etnomusicologia; Universidade Federal da Paraíba; João Pessoa, Paraíba, Brasil.
} sonodadoc@gmail.com 
literatura, análisis de rendimiento de los estudiantes en el proyecto, los datos obtenidos en la primera fase de la investigación y observación participante, entrevistas, técnicas de gráficos y tablas. Se espera que este trabajo contribuirá a la profundización y ampliación de las discusiones en torno de la cuestión de las iniciativas educativas e institucionales para evitar las influencias de su tipo en el rendimiento académico.

Palabras claves: Análisis acústicos, análisis de Presión Sonora, UFPB, rendimiento académico.

\section{O Projeto de Pesquisa}

A audição humana é um dos principais mecanismos para a comunicação e orientação espacial, apesar de utilizada também para apreciação musical, discriminação auditiva, habilidades motoras, vocabulário, raciocínio não verbal, leitura, desenvolvimento do QI, habilidades matemáticas, etc. (FORGEARD, et al., 2008; SCHELLEMBERG, 2004; MORENO, et al., 2008; AMATUCCI; LUPION, 2001; KURRLE; TONIOLO, 2004; MENDONÇA; LEMOS, 2010; GROß, et al., 2010; ANVARI, 2002).

Tal mecanismo é fundamental para interpretação dos ambientes, apesar de suscetível à danos quando submetido a índices elevados de pressão sonora (PEREIRA BRANCO, 2013, p.69; EVEREST, 2001, p.78; HOWARD; ANGUS, 2001, p.68; GONÇALVES, et al, 2009). Exposições estas, capazes de favorecer resultados acadêmicos pouco eficientes em função de características de redução da atenção e dificuldade de concentração, sobretudo acerca dos processos educativos.

Um segundo aspecto preocupante em ambiente escolar relaciona-se com a compreensão da fala em ambientes com acústicas impróprias para processos pedagógicos. Uma vez que o desenvolvimento escolar tem relações diretas com a compreensão da exposição verbal do professor, um contexto acústico da sala que privilegie a compreensão verbal é imprescindível.

Em 2015, o Projeto de Pesquisa: Análise dos Índices de Pressão Sonora no Campus I da Universidade Federal da Paraíba (UFPB), foi aprovado no âmbito do Departamento de Mídias Digitais da Instituição, visando elaborar um quadro situacional das características acústicas de ambientes de interação discente.

Após a primeira etapa do projeto, a qual possibilitou o conhecimento dos índices de pressão sonora em alguns pontos de convivência do Campus I, a segunda etapa será implementada visando análises acústicas dentro de salas de aula, constituindo um conjunto de dados que permita conhecer as influências dos contextos acústicos nos processos pedagógicos de forma mais claras e específica com base na experiência de pesquisa da UFPB em 2015.

Considerando aspectos como níveis legais de pressão sonora, poluição sonora crescente e a vulnerabilidade do ouvido humano frente a elevados índices de SPL (Sound Pressure Level - SPL), conhecer as características acústicas do Campus I da UFPB possibilitou a 
identificação de riscos à audição e possíveis influências negativas no desempenho acadêmico, além de possíveis danos à saúde de servidores da Instituição e do desenvolvimento de pesquisas e testes iniciais no âmbito do controle acústico com base em ações preventivas que garantam a integridade física e intelectual em contextos educacionais.

Com a conclusão da primeira parte da pesquisa, foi possível identificar locais e horários de risco auditivo para seres humanos, além de viabilizar o desenvolvimento e teste de um protótipo eletroacústico de redução da intensidade sonora por controle de fase e inversão de polaridade em ambientes confinados. O desenvolvimento deste protótipo seguirá no sentido de implementar o procedimento em espaço aberto e compreender seu índice de eficiência nestes contextos.

Neste primeira etapa, 06 alunos e 01 técnico de áudio do Departamento compuseram, junto ao coordenador do projeto, a equipe responsável pelo mapeamento em pontos de convivência no Campus I da Instituição. Com a implementação da segunda etapa, espera-se um pequeno, mais imprescindível aumento da equipe, visando resultados mais significativos em âmbitos quantitativos e, principalmente, qualitativos.

Entre os aspectos que justificaram o projeto, salienta-se: a atual recorrência de valores acentuados de SPL no Campus I, responsáveis por incômodos e perdas auditivas (GONÇALVES, et al. 2009); desrespeito aos limiares de pressão sonora legais, favorecendo danos auditivos entre discentes e servidores públicos da Instituição (GONÇALVES, et al. 2009); a importância dos resultados do projeto para soluções em saúde pública com base em medidas preventivas e mudança de comportamentos; mapeamento dos índices de SPL na UFPB, essencial para a compreensão de influências pedagógicas entre servidores e estudantes; desenvolvimento e teste de um protótipo de redução de SPL por inversão de polaridade (BALLOU, 2009. p.57) e cancelamento de fase (WATKINSON, 1998, p.204); envolvimento de graduandos com pesquisa científica, mediante relação com o Programa Institucional de Voluntários de Iniciação Científica da UFPB (PIVIC/ UFPB); maior integração entre ensino, pesquisa e extensão, com desenvolvimentos teórico-práticos desde o início da graduação; impacto direto na produção intelectual e científica do referido Departamento; difusão de atividades de pesquisa em termos quantitativos e qualitativos (QUEIROZ, 2006); encorajamento e aprimoramento da produção bibliográfica discente, etc.

A primeira etapa do projeto foi orientada para análise dos níveis de SPL, visando demonstrar se existe e qual o risco de perdas auditivas por exposição a esses limiares, além de possibilitar o desenvolvimento e teste do protótipo de redução da intensidade sonora em ambiente confinado. Na segunda etapa, ainda por ser desenvolvida, a análise será voltada para 
acústica das salas de aula e níveis de inteligibilidade de exposições verbais em processos pedagógicos.

Com base nos resultados desta primeira parte do projeto, percebeu-se que as influências acústicas no desempenho acadêmico são importantes demais e devem ser amplamente estudadas em função de um conhecimento mais aprofundado e específico da questão.

O desconhecimento da influência de variáveis desse tipo no desempenho pedagógico pode favorecer prejuízos irreversíveis em gerações de estudantes. Dessa forma, a temática parece de importância principal para Instituições e Coordenações Pedagógicas nos mais variados níveis educacionais e em todos os âmbitos de ensino. Por outro lado, quanto mais cedo conhecermos as possíveis influências que a acústica de salas de aula exercem ou podem exercer sobre o desenvolvimento pedagógico e cognitivo de estudantes, menos graves tendem a ser as consequências acadêmicas.

Enquanto delimitação de termos e expressões, visando favorecer compreensões segundo perspectivas adequadas às características e considerações do projeto, alguns pontos foram considerados mais importantes, portanto, destacados.

Inicialmente, a expressão ouvido humano orientada segundo parâmetros sugeridos por Everest (2001, p.41), nos quais são considerados como complementares a estrutura física e a psicológica.

Um segundo aspecto foi a expressão percepção auditiva humana relativa às capacidades auditivas normais para seres humanos adultos, as quais se relacionam com variações entre $20 \mathrm{~Hz}$ e $20 \mathrm{kHz}$ e entre $1 \mathrm{~W} / \mathrm{m}^{2}$ (120dB-A) e $10^{-12} \mathrm{~W} / \mathrm{m}^{2}$ (0dB-A) (EVEREST, 2001, p.41-81; TALBOT-SMITH, 1999, p.1.38-1.43).

Apesar da diversidade de publicações acerca de temas relacionados com ouvido humano, percepção auditiva e amplitude sonora, não foram encontrados estudos anteriores que abordassem análises de SPL no Campus I da UFPB, sugerindo um provável desconhecimento dos riscos para a audição humana, além das possíveis consequências pedagógicas destes contextos acústicos.

Dentre os estudos relacionados com audição humana, perdas auditivas e tecnologias de áudio, foi possível encontrar uma gama de publicações, abrangendo as áreas de acústica, engenharia, tecnologia de áudio (FLETCHER, H.; MUNSON, W.A., 1933; FAHY; WALKER, 2004; EVEREST, 2001; KATZ, 1970; TALBOT-SMITH, 1999; WHITE, 1999; HOWARD; ANGUS, 2001; EARGLE, 2002; FARIA, 2005; VALLE, 2007), medicina e fonoaudiologia (PEREIRA BRANCO, 2013; KNOBEL, 2007; EUGÊNIO; ESCALDA; LEMOS, 2011; GIMENEZ, 2004; CIOQUETA, 2006; GONÇALVES, et al, 2009), música, 
musicologia e comunicação (CHANAN, 1995; MEINTJES, 2003; GARCIA, 2004). Contudo, apesar dos trabalhos mencionados abordarem questões relacionadas ao ouvido ou à percepção auditiva humana, com base nesta revisão bibliográfica, o estudo proposto não parece recorrente na ciência, sobretudo, em função da escassez bibliográfica percebida.

Em termos metodológicos, o projeto contemplou um mapeamento de amplitude ao longo do espectro sonoro audível com medições em Departamentos, Centros e locais de convívio do Campus I da UFPB em dias e horários diferentes para elaboração de tabelas e gráficos indicativos das variações de SPL ao longo do período e dos locais de análise.

Técnicas, procedimentos e práticas qualitativas e quantitativas (BAUER; GASKELL, 2002; QUEIROZ, 2006), como análise bibliográfica e documental, técnicas de entrevistas estruturadas (DUARTE, 2002) e questionários, amostragem, análises e testes acústicos, foram associadas à análises de espectro sonoro, análises comparativas, tabelas e gráficos. Além disso, técnicas de observação participante (SILVA, 2000), precisaram compor as opções metodológicas, sobretudo, em função da elucidação de informações acerca dos ambientes em estudo.

As análises de SPL foram realizadas com medidores de pressão sonora (decibelímetros) em dias e horários diversos para obtenção de média aritmética, favorecendo uma verificação mais precisa das análises de pressão sonora em cada local.

Os relatórios (parciais e final da primeira etapa) empregaram formatação ABNT (NBR), gráficos e análises. Além disso, todos os envolvidos no projeto, principalmente discentes, foram encorajados e orientados no sentido de práticas de publicação científica sobre suas respectivas atividades ao longo do processo de pesquisa.

\section{Conclusão}

A implementação do projeto no Campus I da UFPB possibilitou um levantamento inicial das características acústicas dos locais analisados, sugerindo que problemas de saúde como perdas auditivas, estresse, diminuição da atenção, etc. podem decorrer da exposição aos limiares de pressão verificados nestes ambientes. A segunda etapa do projeto deverá identificar características acústicas das salas de aula que podem contribuir para um baixo desempenho pedagógico discente, em decorrência das dificuldades de compreensão da fala nestes ambientes.

Com o desenvolvimento da segunda parte da pesquisa, provavelmente será possível determinar se ocorrem influências no desempenho pedagógico e cognitivo discente que justifiquem mudanças na forma como se projeta uma sala de aula, além de revelar que tipo de influência sobre os processos educacionais estamos enfrentando no âmbito do corpo discente 
em estudo.

Se os resultados acústicos de ambientes projetados para a educação estão inadequados para sua função principal, a comunidade científica deve conhecer com precisão que tipo de influências são essas, além das opções legais, procedimentais e pedagógicas disponíveis para solucionar tal problemática em um curto espaço de tempo, sobretudo, com vistas à melhorias no processo educacional de forma geral.

Espera-se que esta iniciativa de pesquisa possa alertar a comunidade científica sobre possíveis consequências das características acústicas dos ambientes para processos pedagógicos, além de favorecer o desenvolvimento de soluções coerentes e imediatas para a problemática.

\section{Referências}

AMATUCCI, M.A.F.C.; LUPION, A.S. Das habilidades auditivas de localização, memória e figura-fundo, 2001.

ANVARI, S.H.; TRAINOR, L.J.; WOODSIDE, J.; LEVY, B.A. Relations among musical skills, phonological processing, and early reading ability in preschool children. J Exp Child Psyc. 2002;83(2):111-30. In: EUGÊNIO, Mayra Lopes; ESCALDA, Júlia; LEMOS, Stela Maris Aguiar. Desenvolvimento cognitivo, auditivo e linguístico em crianças expostas à música: produção de conhecimento nacional e internacional. Revista CEFAC. São Paulo: 2011.

BALLOU, Glen. Electroacoustic devices: microphones and loudspeakers. Focal Press, 2009.

BAUER, Martin W.; GASKELL, George. Pesquisa qualitativa com texto, imagem e som: um manual prático. Tradução de Pedrinho A. Guareschi. 5. ed. Petrópolis: Vozes, 2002.

CHANAN, Michael. Repeated takes: a short history of recording and its effects on music. New York: Verso, 1995.

CIOQUETA, Elenara Pilar. Efeito da prática musical no processamento auditivo em escolares de sete a 14 anos de idade. Dissertacão de Mestrado. Programa de Pós-Graduação em Distúrbios da Comunicação Humana, Área de Concentração em Audiologia. Universidade Federal de Santa Maria (UFSM), Brasil. 2006. Orientadora: Profa. Dra. Maristela Julio Costa. DUARTE, Rosália. Pesquisa qualitativa: reflexões sobre o trabalho de campo. Cadernos de Pesquisa. n.115, p.139-154. 2002.

EARGLE, John. Handbook of recording engineering. 4. ed.. Los Angeles: JME Consulting Corporation, 2002.

EUGÊNIO, Mayra Lopes; ESCALDA, Júlia; LEMOS, Stela Maris Aguiar. Desenvolvimento cognitivo, auditivo e linguístico em crianças expostas à música: produção de conhecimento nacional e internacional. Revista CEFAC. São Paulo: 2011. 

e-ISSN 2016/Atual: 2525-7870 | e-ISSN 2015/2016: 2447-018X

EVEREST, F. Alton. Master Handbook of Acoustics. McGraw-Hill Companies Inc., 2001.

FAHY, Frank; WALKER, John. Advanced Applications in Acoustics, Noise and Vibration. Spon Press, 2004.

FARIA, Regis Rossi Alves. Auralização em ambientes audiovisuais imersivos. Tese de Doutorado. Departamento de Engenharia de Sistemas Eletrônicos. Escola Politécnica da Universidade de São Paulo, Brasil. 2001. Orientador: João Antônio Zuffo.

FLETCHER, H.; MUNSON, W.A. Loudness, its definition, measurement and calculation. Journal of the Acoustical Society of America. v.5, p.82-108, 1933.

FORGEARD, M; WINNER, E; NORTON, A.; SCHLAUG, G. Practicing a musical instrument in childhood is associated with enhanced verbal ability e nonverbal reasoning. Plosone Res. 2008; 10(3):1-8. In: EUGÊNIO, Mayra Lopes; ESCALDA, Júlia; LEMOS, Stela Maris Aguiar. Desenvolvimento cognitivo, auditivo e linguístico em crianças expostas à música: produção de conhecimento nacional e internacional. Revista CEFAC. São Paulo: 2011.

GIMENEZ, Tânia Nabas; MEDRANO, Lígia Maria Martins; SANCHEZ, Maura Lígia; CAMARGO, Zuleica. Estudo das funções centrais - duração e frequência - nas alterações vocais. Rev CEFAC, São Paulo, v.6, n.1, 77-82, jan-mar, 2004.

GONÇALVES, Cláudia Giglio de Oliveira; LACERDA, Adriana Bender Moreira; ZOCOLI, Angela Maria Fontana; OLIVA, Flávia Cardoso; ALMEIDA, Suzanne Bettega; IANTAS, Milena Raquel. Percepção e o impacto da música na audição de integrantes de banda militar. Rev Soc Bras Fonoaudiol. 2009;14(3):515- 20.

GROß, W.; LINDEN, U.; OSTERMANN, T. Effects of music therapy in the treatment of children with deleyed speech development: results of a pilot study. BMC Complem Alternative Med. 2010;39(10):3-10. In: EUGÊNIO, Mayra Lopes; ESCALDA, Júlia; LEMOS, Stela Maris Aguiar. Desenvolvimento cognitivo, auditivo e linguístico em crianças expostas à música: produção de conhecimento nacional e internacional. Revista CEFAC. São Paulo: 2011.

HOWARD, David M.; ANGUS, James A.S. Acoustics and psychoacoustics. 2 ed. Oxford: Focal Press, 2001.

KATZ, Mark. Capturing sound: how technology has changed music. University of California Press, Berkeley and Los Angeles, 1970.

KNOBEL, Keila Alessandra Baraldi. Influências do silêncio e da atenção na percepção auditiva: implicação na compreensão do zumbido. Tese de Doutorado em Medicina. Universidade de São Paulo, São Paulo, Brasil. 2007. Orientadora: Dra. Tanit Ganz Sanchez.

KURRLE, M.M; TONIOLO, I.M.F. Musicoterapia nas dificuldades do processamento auditivo. Dissertaçao de Mestrado. Programa de Pós-Graduação em Distúrbios da Comunicação Humana. Departamento de Fonoaudiologia, 2004. In: EUGÊNIO, Mayra Lopes; ESCALDA, Júlia; LEMOS, Stela Maris Aguiar. Desenvolvimento cognitivo, auditivo e linguístico em crianças expostas à música: produção de conhecimento nacional e 
internacional. Revista CEFAC. São Paulo: 2011.

MEINTJES, Louise. Sound of Africa - Making Music Zulu in a South African Studio. Durham \& London: Duke University Press, 2003.

MENDONÇA, J.E; LEMOS, S.M.A. Relações entre prática musical, processamento auditivo e apreciação musical em crianças de cinco anos. Rev ABEM. 2010;23(1):58-66. In: EUGÊNIO, Mayra Lopes; ESCALDA, Júlia; LEMOS, Stela Maris Aguiar. Desenvolvimento cognitivo, auditivo e linguístico em crianças expostas à música: produção de conhecimento nacional e internacional. Revista CEFAC. São Paulo: 2011.

MORENO, S; MARQUES, C; SANTOS, A. SANTOS, M.; CASTRO S.L; BENSSON M. Musical training influences linguistic abilities in 8-year-old children: more evidence for brain plasticity. Cerebral Cortex March Res. 2008; 19(3):712-23. In: EUGÊNIO, Mayra Lopes; ESCALDA, Júlia; LEMOS, Stela Maris Aguiar. Desenvolvimento cognitivo, auditivo e linguístico em crianças expostas à música: produção de conhecimento nacional e internacional. Revista CEFAC. São Paulo: 2011.

PEREIRA BRANCO, Elisabete Rainho Pereira. O ruído nas escolas. Dissertação de Mestrado apresentada à Faculdade de Medicina da Universidade de Coimbra, 2013. Orientador: Isabel Cristina Gaspar Pestana da Lança. Co-Orientador: Dr. António Manuel Pinto Brochado Moreira de Morais

QUEIROZ, Luis Ricardo Silva. Pesquisa Quantitativa e Pesquisa Qualitativa: Perspectivas para o Campo da Etnomusicologia. Claves: Revista do Programa de Pós-Graduação em Música da Universidade Federal da Paraíba, João Pessoa, n. 2, p. 87-98, 2006.

SCHELLEMBERG, E.G; Music lessons enhance IQ. American Psyc Society Res. 2004;15(8):511-4. In: EUGÊNIO, Mayra Lopes; ESCALDA, Júlia; LEMOS, Stela Maris Aguiar. Desenvolvimento congnitivo, auditivo e linguístico em crianças expostas à música: produção de conhecimento nacional e internacional. Revista CEFAC. São Paulo: 2011.

SILVA, Vagner Gonçalves da. Observação participante e escrita etnográfica. In: FONSECA, Maria Nazareth Soares (Org.) Brasil afro-brasileiro. Belo Horizonte: Autêntica, 2000.

TALBOT-SMITH, Michael. Audio engineer's reference Book. 2.ed. Focal Press, 1999.

VALLE, Sólon do. Manual prático de acústica. 2. ed. Rio de Janeiro: Música e Tecnologia, 2007. 355 p.

WHITE, Paul. Creative recording 2: microphones, acoustics, soundproofing and monitoring. London: Sanctuary Publishing Limited. London: 1999.

WATKINSON, John. The art of sound reproduction. Focal Press, 1998. 